\title{
Gastric Outlet Obstruction in the Current Era-A Pictorial Review on Computed Tomography Imaging
}

\author{
Ashita Rastogi1,@ Somesh Singh ${ }^{2}$ Rajanikant Yadav² \\ ${ }^{1}$ Department of Radiodiagnosis, Aster Hospitals, Dubai, \\ United Arab Emirates \\ ${ }^{2}$ Department of Radiodiagnosis, Sanjay Gandhi Postgraduate \\ Institute of Medical Sciences, Lucknow, Uttar Pradesh, India

\begin{abstract}
Address for correspondence Ashita Rastogi, MBBS, DNB, Department of Radiodiagnosis, Aster Hospitals, Near Sharaf DG Mankhool, Kuwait Road, Al Mankhool, Dubai, United Arab Emirates (e-mail: ashitarastogi@hotmail.com).
\end{abstract}

J Gastrointestinal Abdominal Radiol ISGAR 2021;4:139-148.

\begin{abstract}
Keywords

- CT imaging

- CT stomach

- gastric emergencies

- gastric outlet obstruction

Gastric outlet obstruction is a pathophysiological entity characterized by mechanical impediment of gastric emptying, which may occur due to a variety of intrinsic or extrinsic causes affecting the antrum or pylorus or duodenum. The obstruction may be benign or malignant or secondary to a motility disorder. Imaging in gastric outlet obstruction identifies majority of these causes and may indirectly even point toward motility disorders. The advent of computed tomography imaging and its subsequent advances have allowed it to become the mainstay of evaluation of stomach, particularly in gastric outlet obstruction. In this pictorial review, a few causes of gastric outlet obstruction are exhibited.
\end{abstract}

\section{Introduction}

Gastric outlet obstruction (GOO) is a clinical syndrome characterized by mechanical impediment to gastric emptying as result of a benign or malignant disease process involving the antropyloric region of stomach or the duodenum ( Fig. 1) $)^{1,2}$ Various benign and malignant entities may cause GOO, and patients usually present with epigastric pain, nonbilious vomiting, nausea, loss of appetite due to impaired motility and less commonly upper gastrointestinal bleeding. Historically, peptic ulcer disease has been the most common cause of gastric outlet obstruction. With the advent of Helicobacter pylori regimen and proton pump inhibitors, incidence of peptic ulcer disease has significantly reduced and resulted in a change in the etiological profile of GOO. In the present-day scenario, malignancy is the most common cause of GOO.,4

In Western countries, gastric cancer incidence has also significantly reduced due to low prevalence of H. Pylori. In these countries, pancreatic cancer is now the most common cause of GOO. In India and other south Asian countries, gastric cancer remains the most common cause. ${ }^{2}$ Various causes of GOO are depicted in - Fig. 2.

\section{Computed Tomography Protocol}

Traditionally, barium studies were utilized for evaluation of stomach. However, with the advent of computed tomography (CT) imaging, it has become the mainstay of evaluation, since it can image the mural as well as extramural pathologies of the stomach. Further advances in CT technology also allow for virtual endoscopy and 3D rendering of lesions, enhancing the evaluation of gastric disease entities., ${ }^{5,6}$

For an accurate evaluation of the stomach on CT, the factors to be considered are adequate distension of the stomach, apt gastric imaging protocol and appropriate intraluminal contrast agent. ${ }^{5,6}$ In an inadequately distended stomach, disease may be overlooked, or mucosal folds of a collapsed gastric wall may inadvertently be mistaken for disease. published online

March 15, 2021
DOI https://doi.org/

10.1055/s-0041-1725239 ISSN 2581-9933.
(C) 2021. Indian Society of Gastrointestinal and Abdominal Radiology. This is an open access article published by Thieme under the terms of the Creative Commons Attribution-NonDerivative-NonCommercial-License, permitting copying and reproduction so long as the original work is given appropriate credit. Contents may not be used for commercial purposes, or adapted, remixed, transformed or built upon. (https://creativecommons.org/licenses/by-nc-nd/4.0/). Thieme Medical and Scientific Publishers Pvt. Ltd. A-12, 2nd Floor, Sector 2, Noida-201301 UP, India 


\section{Contrast}

Intraluminal contrast: Positive oral contrast, although allows for good gastric distension, has been rendered inappropriate for evaluation of the stomach, since it may not mix uniformly with gastric contents and create pseudolesions. High attenuation of the contrast within the lumen may mask subtle mucosal lesions/thickening/ulcerations, since it obscures the gastric wall enhancement after administration of intravenous

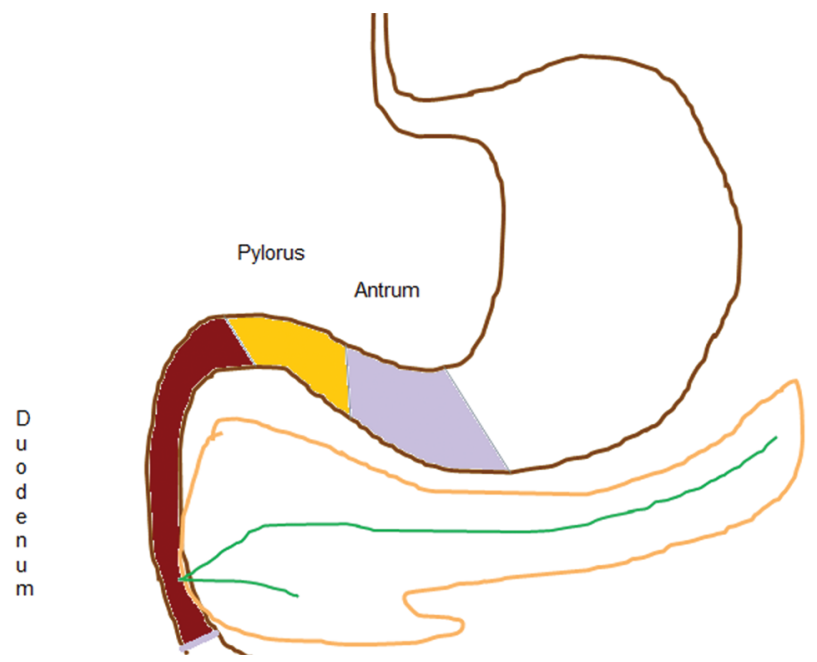

Fig. 1 Relevant gastric anatomy in gastric outlet obstruction-the disease process may be at the level of the antrum, pylorus, or duodenum to result in impediment of gastric emptying and consequent outlet obstruction.
(IV) contrast. In addition, it may interfere with subsequent endoscopic evaluation and make 3D imaging and CT angiography (if performed) postprocessing difficult.

Hence, neutral oral contrast is preferred for evaluation of stomach and gastrointestinal tract (GIT), since it provides excellent contrast between enhancing gastric wall and lumen.

Water is most commonly used medium, because it is well tolerated and provides good contrast in addition to be being cheap and widely available. In most centers, approximately 1 to $1.5 \mathrm{~L}$ of water is administered orally to the patient with three-fourths quantity given 15 to 30 minutes before and rest on the CT table just prior to study.

IV contrast: For IV contrast, 100 to $150 \mathrm{~mL}$ of $350 \mathrm{mg} / \mathrm{mL}$ strength iodinated contrast is administered at approximately $3 \mathrm{~mL} / \mathrm{s}$ followed by a saline flush.

\section{Scanning Protocol}

Conventionally, the stomach is scanned in portal venous phase 70 seconds after IV contrast administration. The protocol is tailored to include a noncontrast phase in cases of gastrointestinal bleeding and may include an arterial phase, with images obtained approximately 25 seconds after beginning of injection to evaluate vessels especially in tumor staging. Usually, $1.25 \mathrm{~mm}$ thick sections which are reformatted at $1 \mathrm{~mm}$ intervals are acceptable for evaluation.

A right lateral decubitus CT may be conducted to obtain adequate distension of antropyloric region and duodenum.

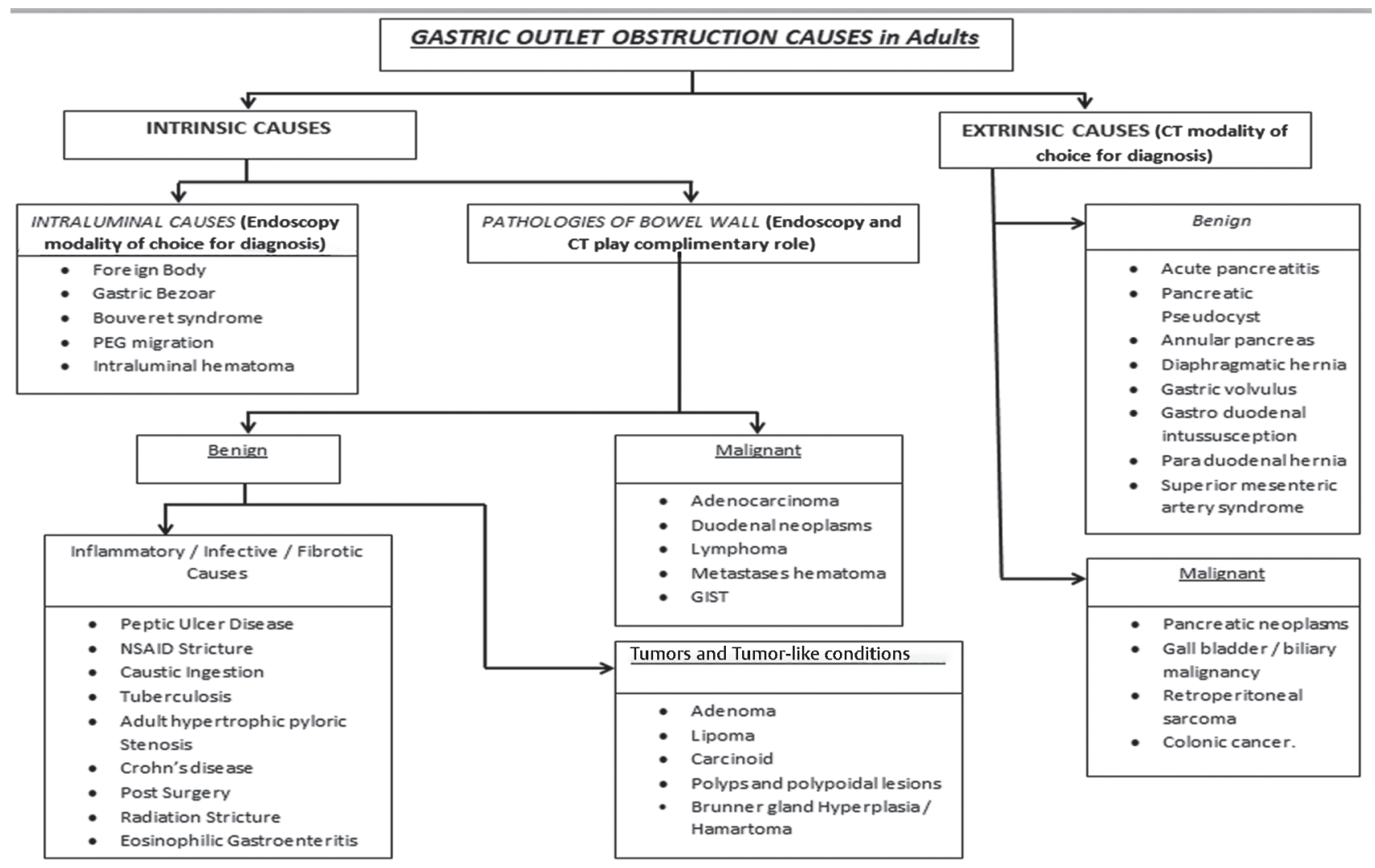

Fig. 2 Causes of gastric outlet obstruction. 
Furthermore, decubitus view may help in delineating relation with pancreatic head in neoplasms.

\section{Pathological Entities Causing Gastric Outlet Obstruction}

\section{Infective/Inflammatory}

\section{Peptic Ulcer Disease}

Peptic ulcer disease (PUD) is an inflammatory response of the gastric mucosa to acid imbalance, which results in erosions and ulcerations. The inflammation may result in edematous thickening of the gastric wall and spasm, with GOO as a consequence. In the setting of chronic disease, obstruction may result from a fibrotic stricture. Ulcers in PUD are usually not demonstrated on routine CT studies, unless they are deep or have penetrated or perforated the gastric wall, or the site of involvement may be identified by perigastric fat stranding (-Fig. 3). The changes of wall thickening and luminal narrowing in outlet obstruction due to PUD may also be indistinguishable from other causes and often require endoscopy and biopsy correlation. ${ }^{1,3}$

\section{Corrosive Stricture}

Corrosive (acids or alkalis) ingestion may be accidental (commonly in children) or suicidal (in adults). Corrosive injuries are more commonly seen in the upper GIT and in the acute setting cause perforation. The healing in these injuries occurs by fibrosis, which can result in stricture of the antrum causing GOO. CT imaging not only helps to identify the location and extent of stricture but also facilitates evaluation of extragastrointestinal involvement ( - Fig. 4). ${ }^{1,7}$

\section{Tuberculosis}

Tubercular affliction of the GIT most often occurs in the ileocecal region. Gastroduodenal tuberculosis (TB) is a rare presentation occurring in 1 to $2 \%$ of abdominal TB cases and more commonly occurs secondary to pulmonary disease. High acid content, rapid gastric transit time, and lack of significant lymphoid tissue may be responsible for relative sparing of gastroduodenal region from tuberculosis. Primary diagnosis of gastroduodenal TB is difficult and may be considered in patients whose disease is refractory to treatment for PUD, in immunocompromised individuals, or in patients with tuberculosis at other sites. ${ }^{1,8}$

Both imaging and endoscopy may reveal nonspecific findings in gastroduodenal TB. Mucosal ulcerations, luminal narrowing, or extrinsic compression may be seen on barium studies, while CT may reveal gastric/duodenal wall thickening, luminal narrowing, and local lymphadenopathy, causing extrinsic compression; the latter two resulting in outlet obstruction ( - Fig. 5). Similar findings may be seen in the small bowel at other sites, particularly ileocecal junction. Usually, lymph nodes are involved in a conglomerate manner and may demonstrate central necrosis on CT. Endoscopy may not reveal any abnormality if the disease is submucosal, or may reveal findings such as nodularity, ulcerations, erythema, or even deformity involving the antropyloric region and duodenum. Most studies report that biopsies may show nonspecific inflammation with diagnosis of gastroduodenal TB made only on postoperative sample, deeming clinical history and radiological search for pulmonary tuberculosis essential for a preoperative diagnosis.

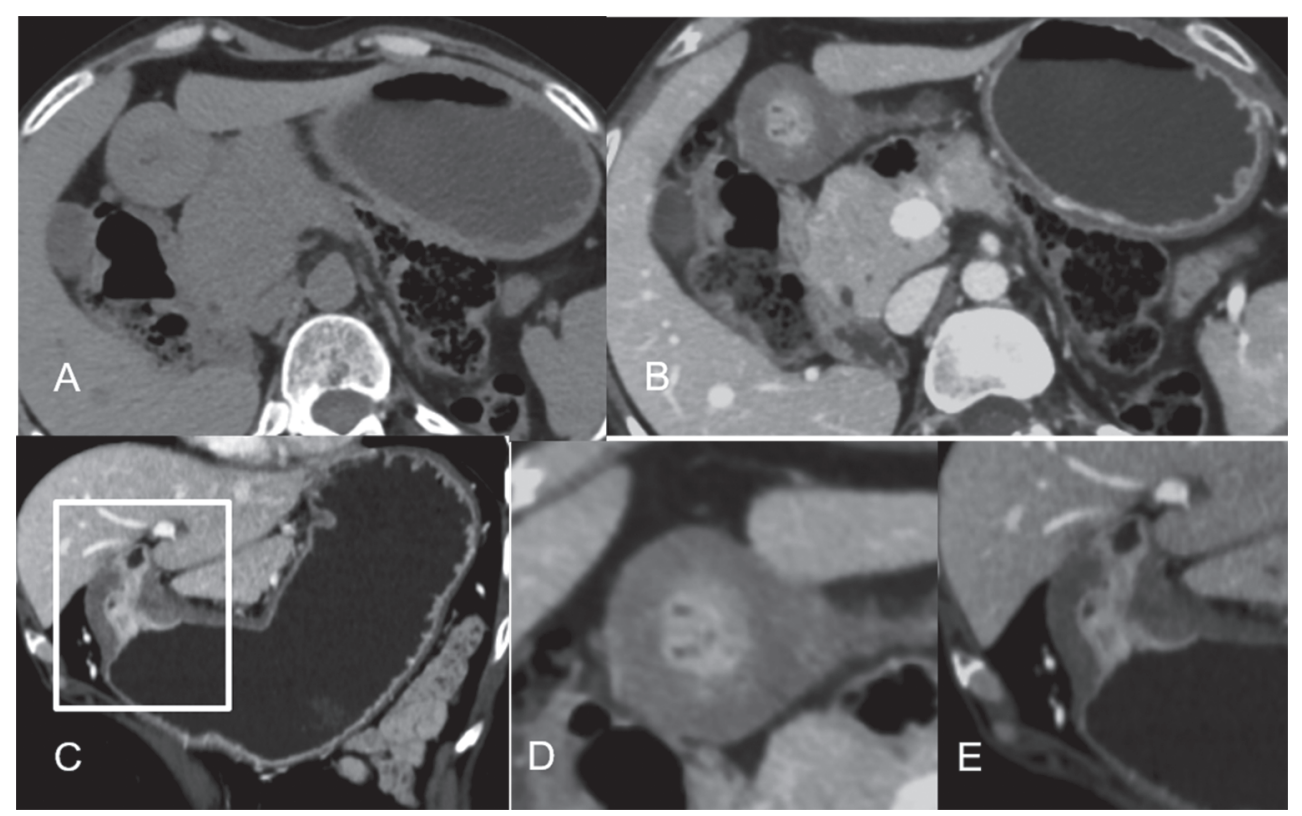

Fig. 3 Gastric outlet obstruction in a patient with known peptic ulcer disease who presented acutely with abdominal pain and vomiting. Axial plain (A) and contrast-enhanced (B) CT image shows low-attenuation wall thickening at the pylorus, resulting in obliteration of the lumen. Oblique coronal (C) shows involvement of entire pyloric length with mucosal defects and air foci within (arrowhead). (D) and (E) show magnified axial and oblique coronal views. Note the maintenance of differentiation between mucosa and submucosa. Subsequent endoscopy confirmed findings of ulceration with biopsy negative for malignancy. 


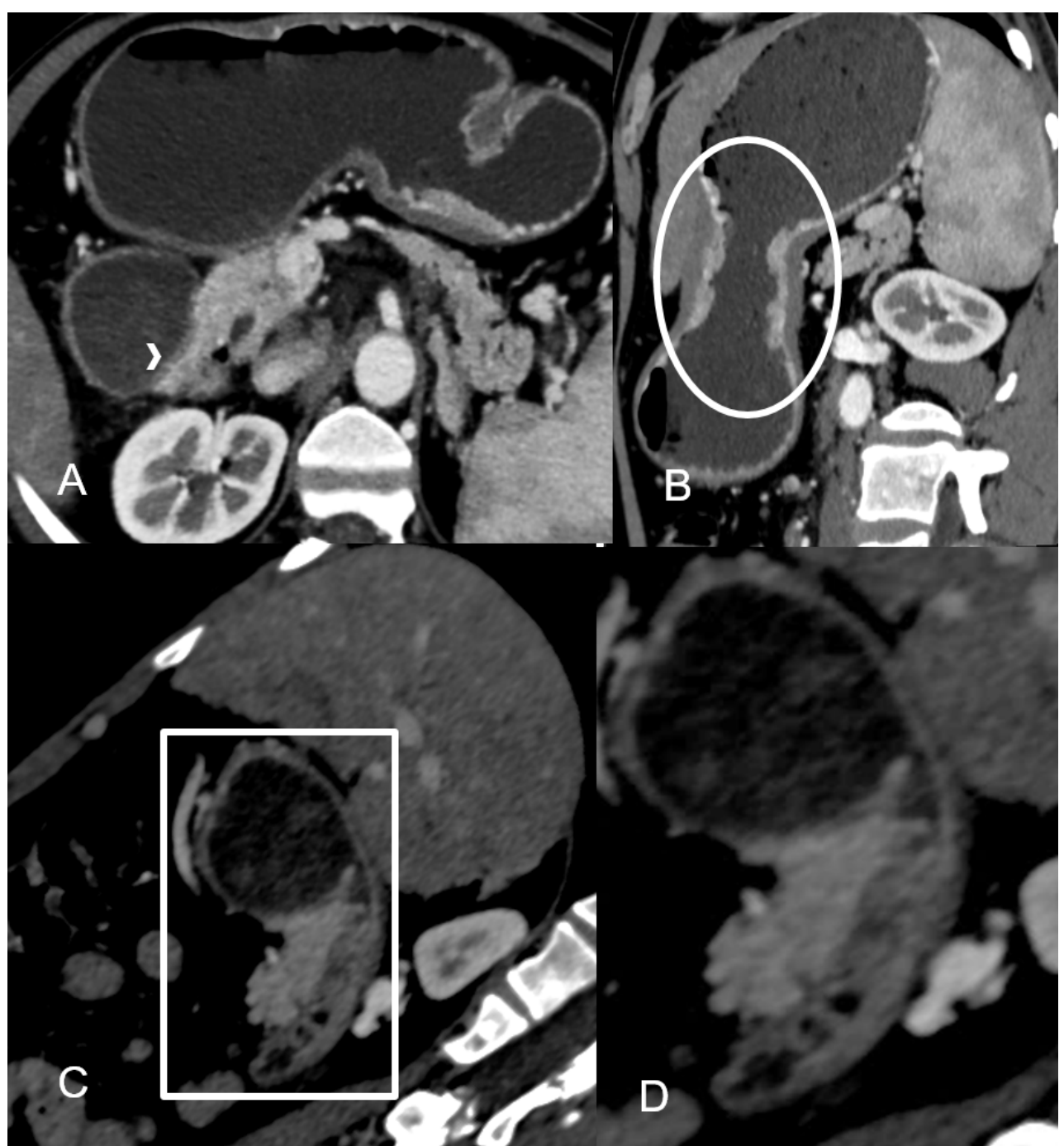

Fig. 4 Gastric outlet obstruction in a patient with history of acid ingestion. Axial (A), oblique sagittal (B) and curved reformat (C) images of antropyloric region of stomach with magnified images of curved reformatted sections (D). There is smooth subtle thickening of the pylorus and D1 segment (arrowhead in A) of duodenum with mild enhancement and narrowing of lumen and over distension of stomach. Note mild waist like narrowing of the body of stomach (encircled in $\mathbf{B}$ ). Note absence of any adjacent fat stranding.

\section{Crohn's Disease}

Crohn's disease is an inflammatory bowel disease that may involve any site of the GIT with predilection for terminal ileum and proximal colon. Gastroduodenal involvement is rare even with disease at other sites, and seldom occurs in isolation. Most commonly the antrum, pylorus, and proximal duodenum are involved in a contiguous manner. ${ }^{9,10}$ Since it involves the GIT in a transmural pattern, abscesses, stenosis, and fistulas are common complications. Primary involvement of the stomach and duodenum usually manifests as ulcer or stricture formation, which may lead to outlet obstruction. Gastroduodenal involvement may also occur secondary to disease in adjacent small bowel of colon loops via a fistulous communication. CT helps to identify the gastric/duodenal inflammation, presence of abscesses and disease at other sites, while CT enterography is a valuable technique for evaluation of location and extent of strictures in CD. Barium studies, particularly double contrast imaging, has conventionally been utilized to assess mucosal disease and demonstrates aphthous ulcerations, cobblestoning, rugal fold thickening, pseudodiverticulae, and strictures, which are also well-visualized at endoscopy (-Fig. 6).

\section{Pancreatitis}

Groove pancreatitis or paraduodenal pancreatitis is a focal form pancreatitis, which may involve the duodenum in the inflammatory process, particularly due to release of exocrine enzymes. There may be resultant duodenal wall edema and scarring along with impaired motility, which may eventually cause outlet obstruction. ${ }^{11}$ 


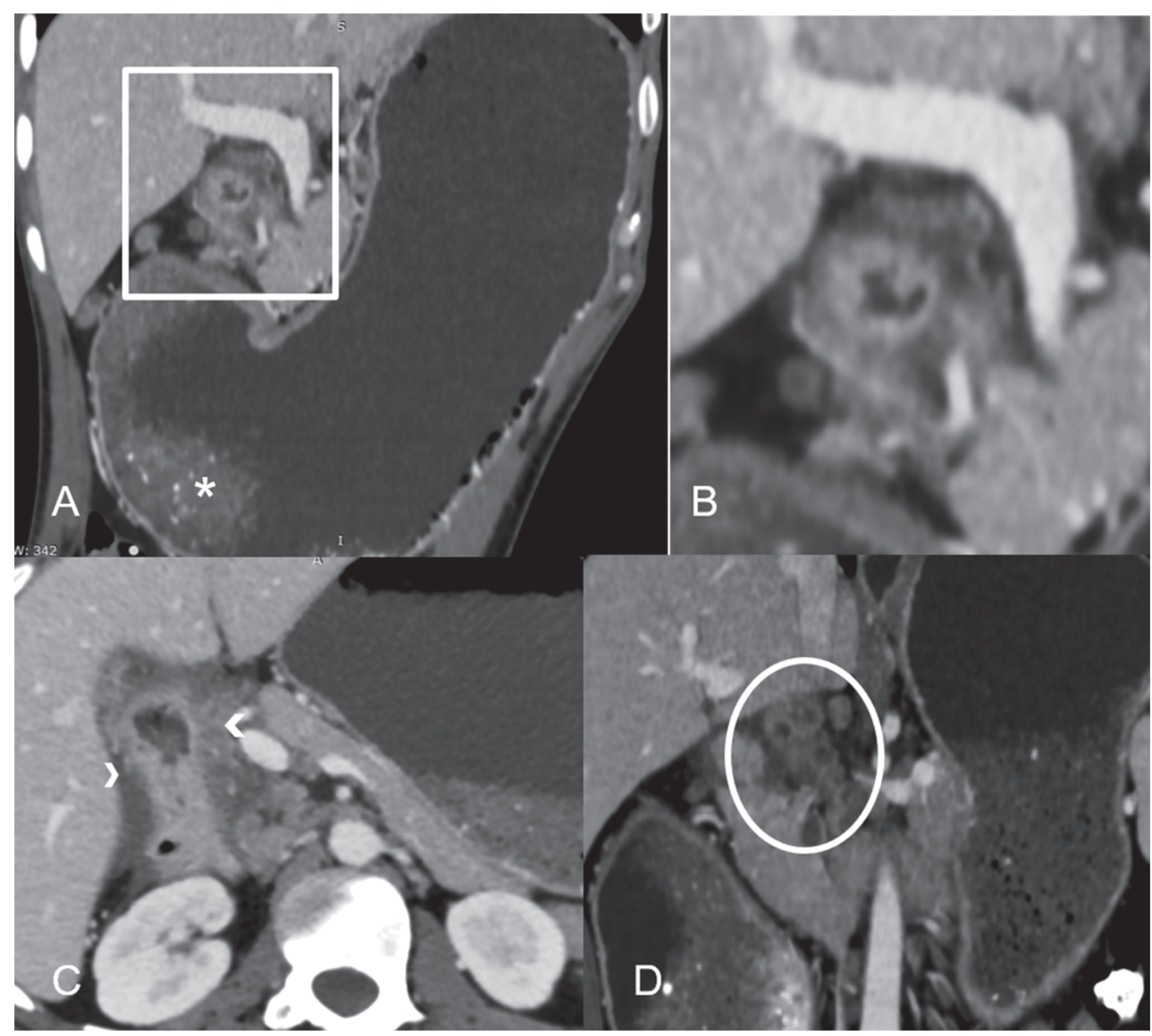

Fig. 5 A 44-year-old male presenting with early satiety and recurrent vomiting and weight loss. Oblique coronal (A), magnified oblique coronal (B) and axial (C) images. Overdistension of stomach with food residue noted (marked as * in A). Irregular circumferential thickening of the pylorus and first part of duodenum seen with extensive surrounding fat stranding (arrowheads). Coronal section (D) shows conglomerate necrotic nodal mass in periportal region. Few discrete necrotic nodes also seen. Endoscopic biopsy was negative for malignancy and fine needle aspiration cytology (FNAC) test of nodal mass showed granulomatous adenitis, positive on GenXprt test.

\section{Neoplasms}

\section{Gastric Adenomyoma}

It is a rare benign subepithelial tumor composed of epithelial elements and smooth muscle proliferation, arising in the stomach or duodenum. ${ }^{12}$ The epithelial component may have differentiated into duodenal or pancreatic tissue. They are most often detected incidentally on CT or endoscopy. Adenomyomas can present with peptic-type ulcers and gastrointestinal bleeding and rarely cause outlet obstruction. The findings on CT and endoscopy are nonspecific and may not even reveal the submucosal lesion. Endoscopic ultrasound (EUS) may help determine the submucosal location of the lesion, as well as cystic change within, and assist in endoscopic resection if feasible. Similar findings of a submucosal lesion with cystic change were seen on CT imaging in our patient (-Fig. 7)

\section{Gastric Polyps}

Polyps are abnormal tissue growths arising from the mucosal lining of any organ. They are of two types-hyperplastic and adenomatous variety. Majority of gastric polyps are hyperplastic polyps arising in a setting of gastritis. Adenomatous polyps are rare and are at higher risk of harboring carcinomatous foci. On CT, hyperplastic polyps are smooth sessile oval lesions, which may occur in clusters, while adenomatous polyps tend to be larger, usually solitary and are more commonly pedunculated. ${ }^{13}$ Polyps, particularly pedunculated ones, have the potential to cause outlet obstruction, since they may invaginate through the lumen ( - Fig. 8 ).

\section{Gastric Adenocarcinoma}

In the recent era, malignancy has superseded peptic ulcer disease as the most common cause of GOO attributable to advances in treatment of the latter. Adenocarcinomas (gastric, duodenal and pancreatic), primary biliary tumors and, less often, lymphomas are the malignant lesions affecting the gastroduodenal region which may cause outlet obstruction. Gastric adenocarcinomas may be early or advanced, based on degree of invasion: early cancers are limited to mucosa or submucosa, while advanced disease invades muscularis propria. ${ }^{3,5}$ To identify gastric cancer, particularly in early stage on $\mathrm{CT}$, adequate distension of stomach is essential. Appearances on CT vary from focal or diffuse mural thickening, which may be nodular and irregular to ulceration with abrupt cutoff of gastric folds or irregular base to polypoidal mass. Local lymphadenopathy and 


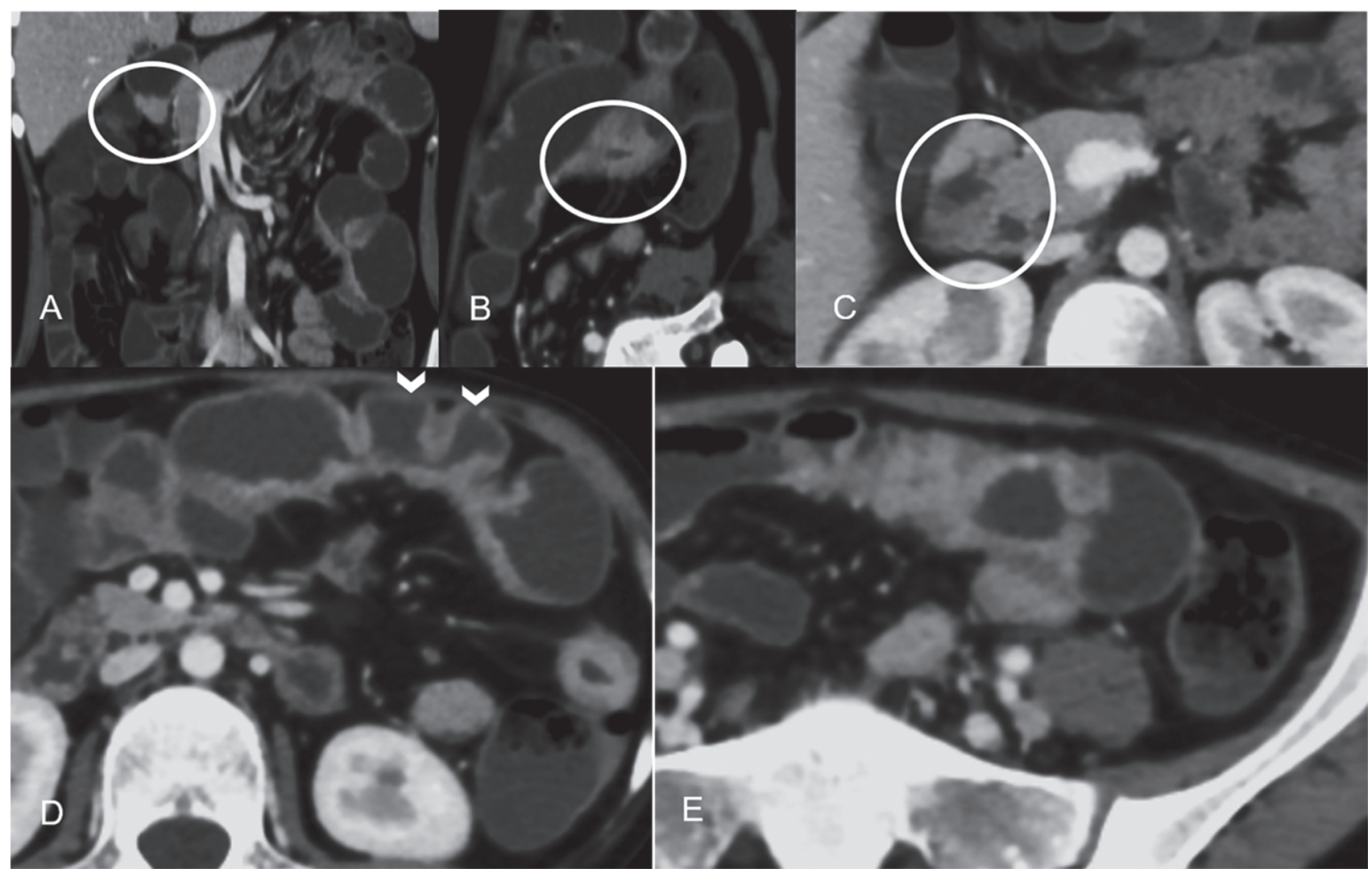

Fig. 6 A 30-year-old patient with recurrent pain abdomen and vomiting. Postcontrast coronal (A), curved reformat (B) and axial (C) images of duodenum shows asymmetric circumferential thickening of second part of duodenum (encircled) with mural stratification, seen better on curved reformat image. Further axial cuts through lower abdomen (D and E) show long-segment thickening distal ileal loops. Note predominant involvement of mesenteric border (D) with pseudosacculations (arrowheads).

metastatic disease at other sites are findings, which may help differentiate malignancy from other causes of outlet obstruction (-Fig. 9).

Duodenal adenocarcinomas may also present with outlet obstruction, particularly when involving the first/second part. CT characteristics of malignant involvement of the duodenum include irregular, eccentric, or circumferential wall thickening with luminal narrowing or even a heterogeneous-enhancing mass.

\section{Lymphoma}

GIT is the most common site of involvement by extranodal lymphoma, with half of them occurring in the stomach. Most gastric lymphomas are nonHodgkin lymphomas of B cell origin and may range from indolent low-grade, mucosa-associated lymphoid tissue (MALT) to aggressive diffuse large B cell lymphomas (DLBCLs). MALT lymphomas are known to be associated with $H$. pylori gastritis, with the inflammatory response acting as a precursor. As for DLBCL, it is thought that it may originate from the low-grade MALT lymphomas or arise de novo.

Gastric lymphomas may present as ulcerations or diffuse infiltration or polypoidal mass or multiple submucosal nodules. CT aids in identifying the lesions as well as in staging the disease in chest and abdomen. It may be seen a focal or diffuse gastric wall thickening, focal or diffuse enlargement of gastric folds (indicating submucosal spread), polypoidal intraluminal mass, or multiple variable-sized submucosal nodules. Features which help differentiate the diffuse infiltrating form from gastric adenocarcinoma include involvement of more than one region of stomach and lymphadenopathy extending below renal hila. Outlet obstruction is a less common feature of lymphoma, since it is a "soft tumor." Infiltration of adjacent organs may be seen in the high-grade lymphomas. ${ }^{5,6}$

\section{Congenital Lesions \\ Annular Pancreas}

Annular pancreas is a congenital anomaly which may have its initial presentation in adulthood. Developmentally, pancreas arises from a ventral and dorsal bud. Failure of rotation of the ventral bud results in pancreatic tissue encircling the duodenum. Annular pancreas is a cause for duodenal obstruction observed in the pediatric age group. In adults, obstruction may result secondary to acute or chronic pancreatitis in the tissue surrounding the duodenum or in a setting of peptic 


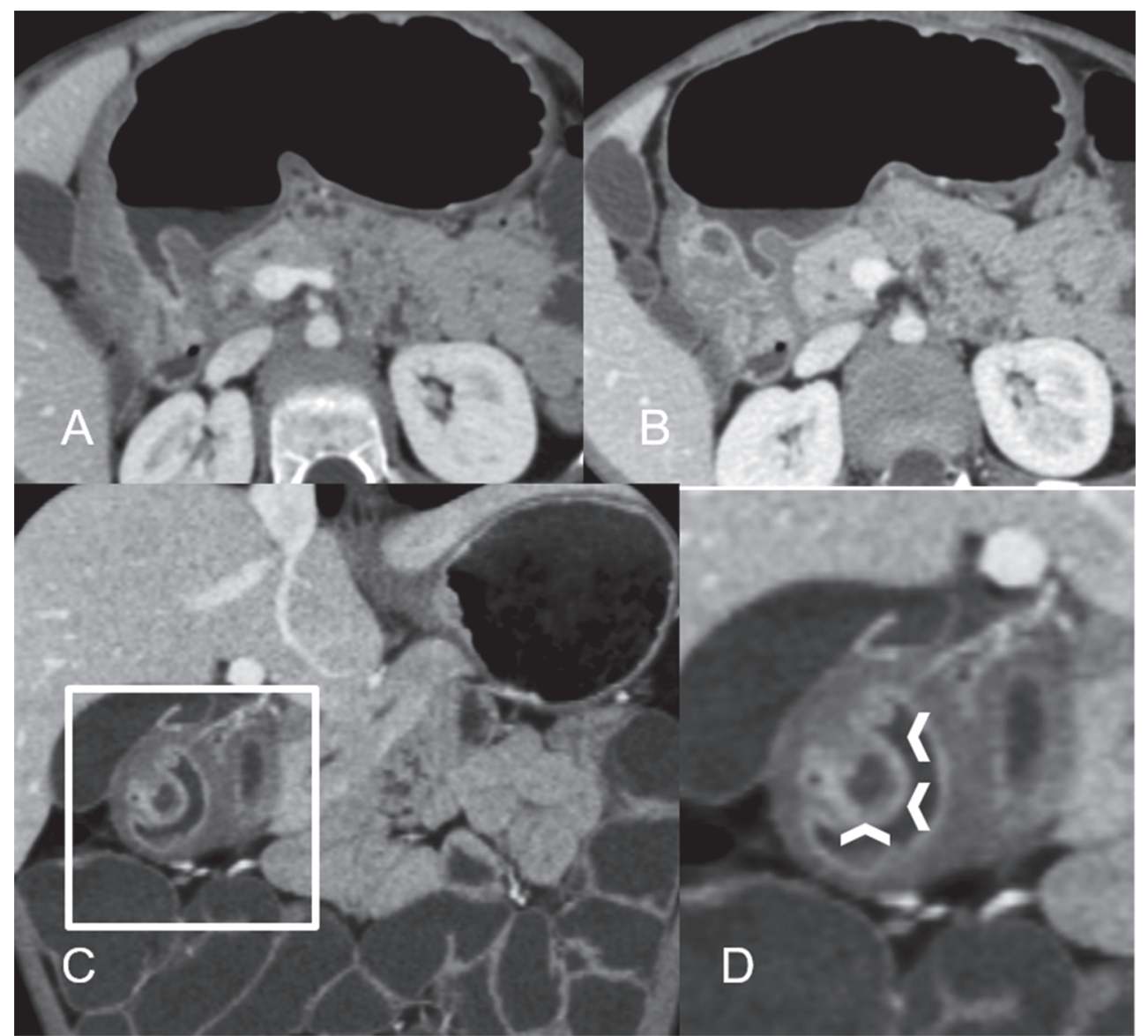

Fig. 7 A 17-year-old patient with recurrent epigastric pain and vomiting. Axial (A and B), coronal (C), and magnified coronal (D) computed tomography images show a well-defined submucosal lesion showing cystic appearance in pyloric region with diffuse edema of pylorus. Note normal enhancing mucosa marked by arrowheads in (D). Repeated endoscopic biopsy showed no tumor and patient underwent distal gastrectomy in view of nonresolving symptoms. Histopathology showed gastric adenoma with heterotopic pancreatic rests.

ulcer disease, which has a known association with annular pancreas..$^{14}$ Biliary obstruction may also be seen in these patients in addition to GOO. ${ }^{15}$

Barium reveals the characteristic "double bubble" sign in the pediatric age group. CT and magnetic resonance cholangiopancreatography (MRCP) may help identify pancreatic tissue encircling the duodenum as well as features of pancreatitis if present. Should CT be performed in pediatric population, it may be useful to administer negative oral contrast and image in the arterial phase to identify enhancing pancreatic tissue around the duodenum, since children have paucity of intra-abdominal fat.

\section{Miscellaneous}

\section{Adult Hypertrophic Pyloric Stenosis}

Hypertrophic pyloric stenosis is typically a disease of neonates and infants and is a rare entity in adults. It has been classified into three types by Danikas et al as follows: Type 1: infantile adult hypertrophic pyloric stenosis (HPS) diagnosed at a late stage wherein history of symptoms in infancy is present, Type 2: most common type which occurs secondary to other underlying gastrointestinal pathologies, such as hiatus hernia, malignancy, and certain inflammatory diseases, and Type 3 : primary idiopathic variety.$^{16} \mathrm{~A}$ more widely accepted classification is into primary and secondary types as reported by Zarineh et al. ${ }^{17}$ When no underlying pathology can be found, it is classified as primary/idiopathic, and in the presence of an underlying disease such as malignancy, postoperative adhesions, fibrosis consequent to healing of ulcers, etc., it is called as secondary HPS. Patients may present with epigastric pain and distension, which is relieved by vomiting.

Imaging in adult HPS may or may not reveal any abnormality in the form of gastric antral/pyloric wall thickening, which is considered abnormal if it is persistent with thickening $>12 \mathrm{~mm}$. CT imaging may also help to identify secondary causes such as hernia, inflammatory changes, or malignancy. A more reliable sign ${ }^{16-18}$ to suggest hypertrophic pyloric stenosis is the "cervix sign," which is seen on endoscopy and refers to a fixed smooth narrowing of the pylorus that persists even after anticholinergic administration ( - Fig. 10).

\section{Gastric Volvulus}

It is the twisting of stomach which may result in outlet obstruction or ischemia and perforation, as is seen with other 


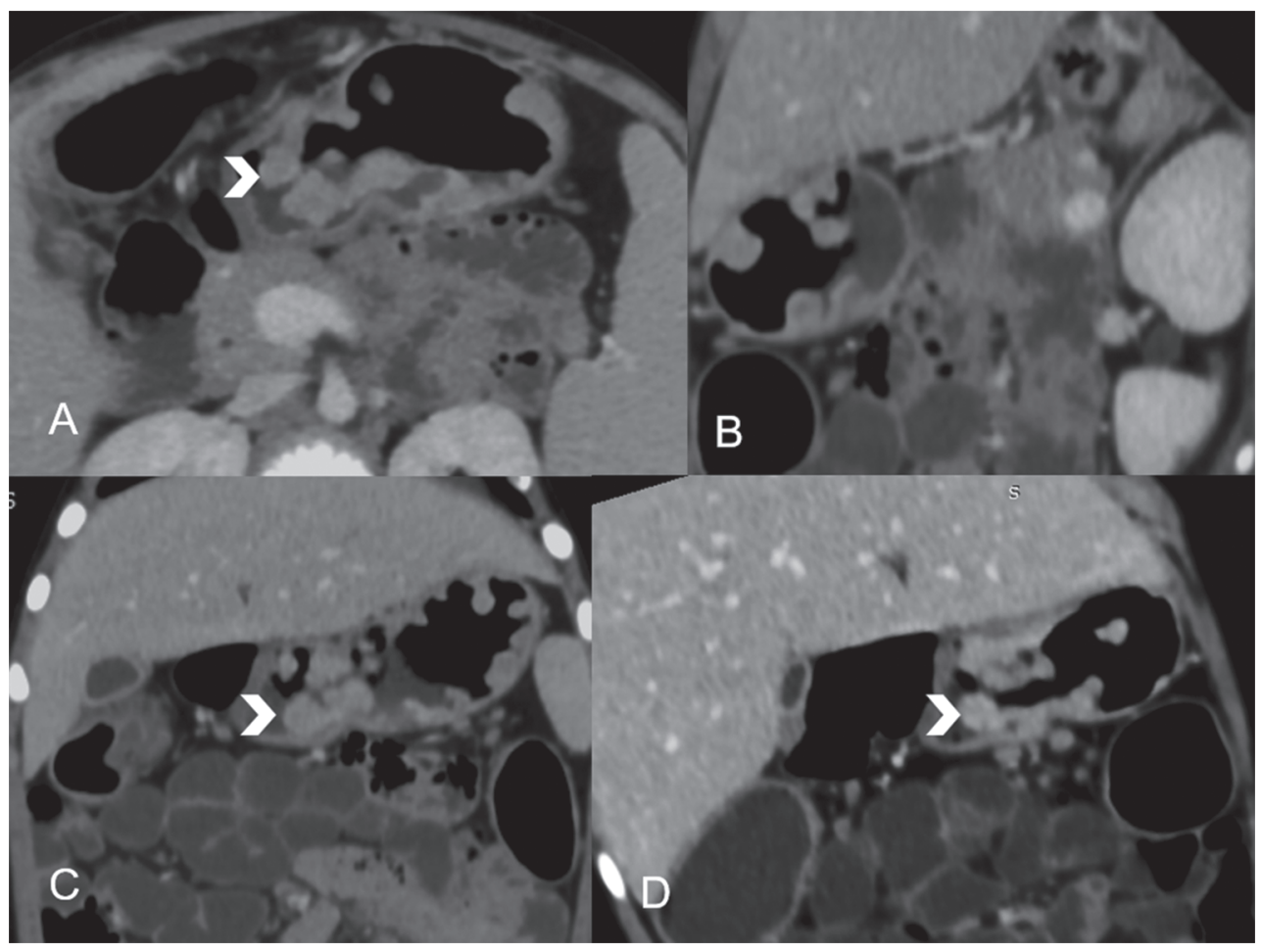

Fig. 8 A 41-year-old patient with intermittent epigastric pain and vomiting. Axial (A), sagittal (B), coronal and oblique coronal (C and D) computed tomography images show multiple homogeneously enhancing polypoidal lesions (many sessile, few pedunculated) involving all regions of stomach including pylorus (arrowheads). Endoscopy and biopsy were performed which showed hyperplastic polyps and isolation of Helicobacter pylori. Final diagnosis: H. pylori related chronic gastritis with multiple hyperplastic polyps.

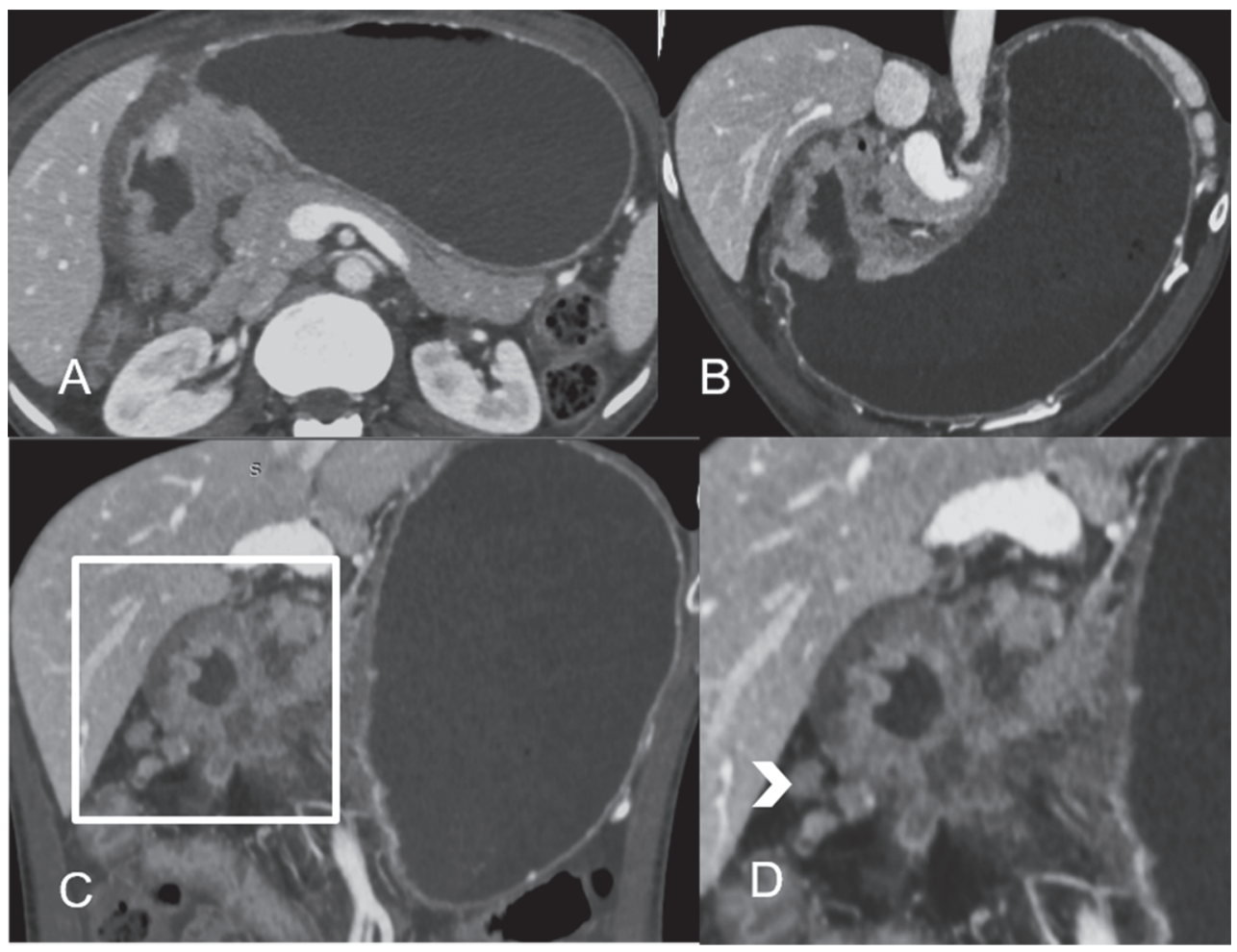

Fig. 9 Patient with epigastric pain and vomiting. Axial (A), oblique coronal (B), coronal (C) and magnified coronal (D) computed tomography (CT) images. CT scan shows irregular enhancing wall thickening in the antropyloric region with luminal narrowing. Perigastric fat stranding and enhancing perigastric lymph nodes (arrowhead in $\mathbf{D}$ ) are also seen. Final diagnosis: gastric adenocarcinoma. 


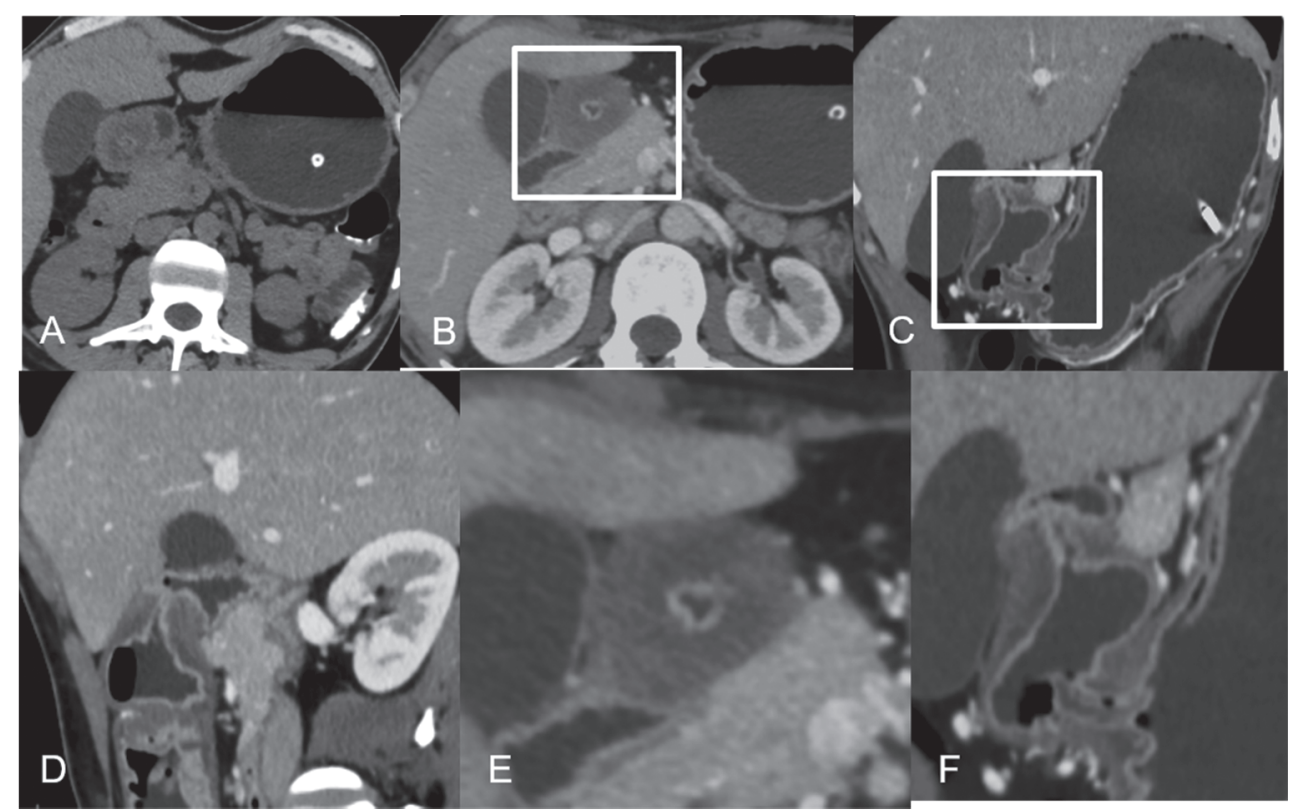

Fig. 10 A 29-year-old patient with recurrent vomiting, weight loss, and epigastric pain. Axial plain (A) and contrast-enhanced (B) computed tomography image shows low-attenuation wall thickening at the pylorus with obliteration of the lumen. Oblique coronal (C) and coronal (D) images show involvement of entire pyloric length. $(\mathbf{E})$ and $(\mathbf{F})$ show magnified axial and oblique coronal views. There is maintenance of differentiation between mucosa and submucosa. Endoscopy showed no ulceration. Biopsy showed presence of $\mathrm{H}$. pylori. Final diagnosis: hypertrophic pyloric stenosis secondary to $H$. pylori-related gastritis.

hollow viscus. Classical presentation may be seen with sudden onset epigastric pain, intractable retching without vomiting, and inability to pass a nasogastric tube, which is known as the Borchardt's triad. Gastric volvulus is commonly associated with hiatus hernias, particularly paraesophageal type. Two subtypes have been described in literature: organoaxial and mesenteroaxial. Organoaxial volvulus occurs when stomach rotates along its long axis, resulting in the antrum moving anterosuperiorly and the fundus rotating posteroinferiorly. The greater curvature lies superior to the lesser curvature in this type, while the relation of the antrum to the gastroesophageal junction is retained. The stomach rotates along its short axis in mesenteroaxial volvulus, resulting in the antrum moving above the gastroesophageal junction. ${ }^{3,4}$

CT findings in gastric volvulus demonstrate the rotation of the stomach with abnormal location and obstruction of the gastric outlet, wall thickening, perigastric fat stranding, and nonpassage of oral contrast. Any hiatus hernia will also be seen in these cases, as herniation of abdominal contents through a widened esophageal hiatus of the diaphragm.

\section{Bezoar/Foreign Body}

Bezoars, although commoner cause of small bowel obstruction, may sometimes result in GOO, particularly in those with impaired gastric motility. These are commonly of two typesphytobezoars composed of fruit and vegetable matter and trichobezoars composed of hair. ${ }^{3,4}$ On CT, a mottled appearance is seen caused by air bubbles retained in interstices of the filling defect within the gastric lumen in addition to feature of obstruction. Another cause of outlet obstruction due to foreign body may be seen in body packers, who may ingest packs of illicit drugs. ${ }^{11}$

\section{latrogenic}

Gastric banding is a widely performed bariatric surgery wherein a silicone band is placed around the stomach approximately $2 \mathrm{~cm}$ distal to the gastroesophageal junction to decrease the capacity of gastric lumen. Accidental slippage of the band to a more distal location may result in features of outlet obstruction. The position of the band may be determined on the topogram or a coronal reformatted image on CT. The band forming a more obtuse angle with the long axis of spine or seen as a complete ring in a horizontal lie with no overlap of its anterior and posterior aspects indicates slippage. ${ }^{3,4}$

\section{Conclusion}

Most patients with GOO present with classical symptoms of nausea and vomiting and bloating with pathologies, affecting the distal stomach or duodenum. In the appropriate clinical setting, CT imaging distinguishes majority of the causes, including intrinsic and extrinsic causes as well as those involving the stomach or the duodenum or surrounding organs and has clearly superseded the traditional barium studies.

\section{Conflict of Interest}

None declared. 


\section{References}

1 Appasani S, Kochhar S, Nagi B, Gupta V, Kochhar R. Benign gastric outlet obstruction-spectrum and management. Trop Gastroenterol 2011;32(4):259-266

2 Godadevi TR, Reddy RA. A clinical study and management of gastric outlet obstruction in adults. Int J Sci Stud 2016;4(6):104-108

3 Guniganti P, Bradenham CH, Raptis C, Menias CO, Mellnick VM. CT of gastric emergencies. Radiographics 2015;35(7): 1909-1921

4 Sunnapwar A, Ojili V, Katre R, Shah H, Nagar A. Multimodality imaging of adult gastric emergencies: a pictorial review. Indian J Radiol Imaging 2017;27(1):13-22

5 Nagpal P, Prakash A, Pradhan G, et al. MDCT imaging of the stomach: advances and applications. Br J Radiol 2017;90(1069): 20160412

6 Horton KM, Fishman EK. Current role of CT in imaging of the stomach. Radiographics 2003;23(1):75-87

7 Kamat R, Gupta P, Reddy YR, Kochhar S, Nagi B, Kochhar R. Corrosive injuries of the upper gastrointestinal tract: a pictorial review of the imaging features. Indian J Radiol Imaging 2019;29(1):6-13

8 Udgirkar S, Surude R, Zanwar V, Chandnani S, Contractor Q Rathi P. Gastroduodenal tuberculosis: a case series and review of literature. Clin Med Insights Gastroenterol 2018;11:1179552218790566

9 Schwartzberg DM, Brandstetter S, Grucela AL. Crohn's disease of the esophagus, duodenum, and stomach. Clin Colon Rectal Surg 2019;32(4):231-242
10 Kefalas CH. Gastroduodenal Crohn's disease. Baylor University Medical Center Proceedings 2003;16(2):147-151

11 Tentugal C, Silva L, Brito J, Soares C, Castro M, Aleixo F. The way to "GOO": mechanisms leading to gastric outlet obstruction and corresponding imaging findings. Paper presented at: European Congress of Radiology, Vienna, Austria; 2013.

12 Duran Álvarez MA, Gómez López JR, Guerra Garijo T. Gastric adenomyoma: The unexpected mimicker. GE Port J Gastroenterol 2017;24(4):198-202

13 Ba-Ssalamah A, Prokop M, Uffmann M, Pokieser P, Teleky B, Lechner G. Dedicated multidetector CT of the stomach: spectrum of diseases. Radiographics 2003;23(3):625-644

14 Sandrasegaran K, Patel A, Fogel EL, Zyromski NJ, Pitt HA. Annular pancreas in adults. AJR Am J Roentgenol 2009;193(2):455-460

15 Kumar A, Agarwal M, Malviya N. Chronic pancreatitis in annular pancreas, presenting with gastric outlet obstruction and bile duct stricture. Journal of Case Reports. 2020;10(2):120-123

16 Hassan SM, Mubarik A, Muddassir S, Haq F. Adult idiopathic hypertrophic pyloric stenosis - a common presentation with an uncommon diagnosis. J Community Hosp Intern Med Perspect 2018;8(2):64-67

17 Zarineh A, Leon ME, Saad RS, Silverman JF. Idiopathic hypertrophic pyloric stenosis in an adult, a potential mimic of gastric carcinoma. Pathol Res Int 2010;2010:614280

18 Lin HP, Lin YC, Kuo CY. Adult idiopathic hypertrophic pyloric stenosis. J Formos Med Assoc 2015;114(7):659-662 